\title{
Health service delivery models for elderly people: A systematic review
}

\author{
Ali Reza Kalantari ${ }^{1}$, Mohammad Hossein Mehrolhassani ${ }^{2}$, Mohsen Shati ${ }^{3}$, Reza Dehnavieh*4 ${ }^{*}$ \\ Received: 29 May 2020 \\ Published: 10 Feb 2021
}

\section{Abstract}

Background: Current Health care delivery systems are not effective for the elderly. Countries with high elderly populations are expected to design special models to serve their elderly population. The aim of this study is to investigate the models of health care delivery to the elderly in different countries.

Methods: The present study is a systematic review based on PRISMA standard guidelines. The search for related studies was conducted in electronic databases (Cochran Library, Scopus, PubMed, Embase, Web of Science) and the Google Scholar search engine without time limits until May 2019. Keywords were extracted based on MeSH strategies. At first, 16243 articles were found. After the screening phase (elimination of duplicated articles, title screening, abstract screening, and full-text screening) 19 articles remained. Two articles deleted after text appraisal using the CASP checklist. In the next stage, after reviewing the gray literature and reviewing the references of remaining articles, three new articles were added (Included studies $=20$ ).

Results: Twenty articles (models) corresponding to the study objectives were finally extracted. These models are limited to nine countries and most have local scopes. These models mainly use a case manager, an intra- or inter-disciplinary team, and an elderly assessment tool in their structure. In addition to the use of an information system, these models provide a wide range of services to the elderly.

Conclusion: Most of the models mentioned are local models. Smaller models to become applicable at the national level, they need to be reviewed and evaluated by policymakers and experts. Given the inefficiency of current systems in providing services to the elderly, it is recommended that countries use an integrated model of health care provision for the elderly.

Keywords: Aging, Elderly, Integrated care, Health care delivery, Financing

Conflicts of Interest: None declared

Funding: Kerman University of Medical Sciences. ((Ethics code: IR.KMU.REC.1399.077)

\section{*This work has been published under CC BY-NC-SA 1.0 license. \\ Copyright $\odot$ Iran University of Medical Sciences}

Cite this article as: Kalantari AR, Mehrolhassani MH, Shati M, Dehnavieh R. Health service delivery models for elderly people: A systematic review. Med J Islam Repub Iran. 2021 (10 Feb);35:21. https://doi.org/10.47176/mjiri.35.21

\section{Introduction}

Increase in the quality of health services along with the increasing access to services has led to an increase in the quality of life and life expectancy of people. This, along with declining birth rates, has led to the aging of the world population. According to the World Health Organization in 2000 , there were 600 million elderly people in the world, which is estimated to increase two times by 2025 and is projected to more than three time by 2050 (1). Currently,

Corresponding author: Dr Reza Dehnavieh, r_dehnavi@kmu.ac.ir

1. Department of Health Management, Policy and Economics, Faculty of Management and Medical Information, Kerman University of Medical Sciences, Kerman, Iran

2. Health Services Management Research Center, Institute for Futures Studies in Health, Kerman University of Medical Sciences, Kerman, Iran

3. Mental Health Research Center, Tehran Institute of Psychiatry-School of Behavioral Sciences and Mental Health, Iran University of Medical Sciences, Tehran, Iran

4. Health Foresight and Innovation Research Center, Institute for Futures Studies in Health, Kerman University of Medical Sciences, Kerman, Iran various countries including Japan, the United States, Sweden, Canada, and Germany are facing the problem of aging, and the population of other countries is aging as well (2). This subject can be a major threat to the future of any country as it can lead to a decrease in the young workforce and an increase in the elderly and the infirm.

Elderly people suffer from age-related problems in different functional dimensions such as in physical, mental,

$\uparrow$ What is "already known" in this topic:

The elderly population of the world is increasing. Countries with high elderly populations are likely to use special models to deliver services to the elderly.

\section{$\rightarrow$ What this article adds:}

This study collected and compared different Health service delivery models for elderly people. Comparing this information gives researchers and policymakers a comprehensive view of service delivery models for the elderly people. 
and social domains, and may be exposed to serious problems such as falls, hospitalizations, disability, and death in the future (3). Among elderly patients, weakness, functional and cognitive decline, loss of independence, use of multiple medications, and coexistence of morbidities are common (4). As these diseases and complications are rarely treated and are the cause of many disabilities and reduced quality of life in the elderly, they play an important role in the use of care and health services $(5,6)$. Increase in the use of services leads to an increase in health services expenditures for the elderly. In addition, the quality of treatment for this population has often been sub-optimal, which can lead to poor outcomes in their health and welfare $(7,8)$.

Overall, the current healthcare delivery system for the elderly is unsuitable due to disintegration and weak coordination among different healthcare specialists. In addition, advanced health information technologies that are facilitators for Health service delivery for elderly people are not widely used $(9,10)$. Moreover, due to regress in the functional dimension and also the existing social disorders in the elderly such as loss of social support, pecuniary constraints, and lack of proper housing, providing traditional medical services is no longer sufficient $(11,12)$. In addition to adequate health care, the elderly need an extensive range of supportive and community services.

Given the importance of quality and comprehensiveness of services as well as the high cost of providing services to the elderly, countries with high elderly populations are expected to have designed unique systems and models for delivery of services to the elderly. Examining these systems is of utmost importance as it can provide other countries with useful experience in this respect. Considering the importance of this issue, the aim of this study is the examination and comparison of health care delivery models for the elderly in different countries.

\section{Methods}

The present study is a systematic review based on the PRISMA standard guidelines. In order to find the answers to the study questions, articles published on the subject of "Health service delivery models for elderly people" were searched in accredited English-language databases without date limits up to 2019.

\section{Search strategy}

The search for related studies was conducted in international electronic databases (Cochran Library, Scopus, PubMed, Embase, and Web of Sciences) without any time limit until May 2019. Keywords were extracted based on MeSH strategies. In this study, three groups of keywords were used:

1. Aging, aged, old, senior, geriatric, frail elderly, older adult, elder. 2. Model, framework, mechanism, theory, pattern, structure, platform. 3. Delivery of health care, health care delivery, healthcare delivery, health service provision.

In order to conduct a comprehensive search, using the operators of the mentioned databases, the combination of the above three groups was searched in the titles, keywords, and abstracts of articles. The search strategy used in the databases was as follows: (aging OR aged OR old OR senior
OR Geriatric OR "frail elderly" OR "older adult" OR elder) AND (model OR framework OR mechanism OR theory OR pattern OR structure OR platform) AND ("delivery of health care" OR "health care delivery" OR "healthcare delivery" OR "health service provision")

In order to improve the quality of the search and to find more studies, the references of the selected articles were also examined.

In order to improve the quality of the search and to find more studies, the Google, Google Scholar search engine and the references of the selected articles were also examined (Fig. 1).

\section{Data Collection}

The selected articles were imported into EndNote software. After removing duplicate titles, the remaining titles, abstracts, and fulltext of the articles were independently reviewed and screened by two of the authors (AR.K and MH. $\mathrm{MH}$ ). Another author (R.D) also reviewed the results, and instances of difference between the two reviews were resolved through discussion. The process used to identify relevant articles is shown in Figure 1.

\section{Inclusion and exclusion criteria}

In order to achieve the research goal, only articles, including health service delivery models for the elderly, were examined. Therefore, the inclusion criteria were: 1 . Articles in English language 2. Articles published until May 2019 (in order to examine the most recent studies, the researchers searched the mentioned databases again on April 6, 2020). 3 . Original articles and conference reports. Exclusion criteria included: 1. Models designed for a specific disease. 2. Models designed for the emergency department. 3. Models designed for nursing homes, systematic review articles, letters to the editors, and editorials were also eliminated from the study.

\section{Quality assessment}

The critical appraisal skills program (CASP) checklist will be used to evaluate the quality of the included article. The CASP checklist comprises 10 questions that can be answered: yes/can't tell/no ('yes' indicates a positive score) (13). The maximum score was 10, and the minimum acceptable score was 7 . Two authors will evaluate the quality of the articles separately. Disagreements between the two authors were resolved by conversation. If necessary, the third author (R.D) were asked to judge.

\section{Data extraction}

The authors reviewed the final articles line-by-line and extracted key points of the models.

At first, it was tried to extract the main contents of the models (findings) based on the health system functions. No model was clearly built on these functions; therefore, the authors tried to extract key points of the models in accordance with the health system functions. Team/team members, case manager and information system (equivalent to resource generation function), services and setting (equivalent to service delivery function), financing (financing 


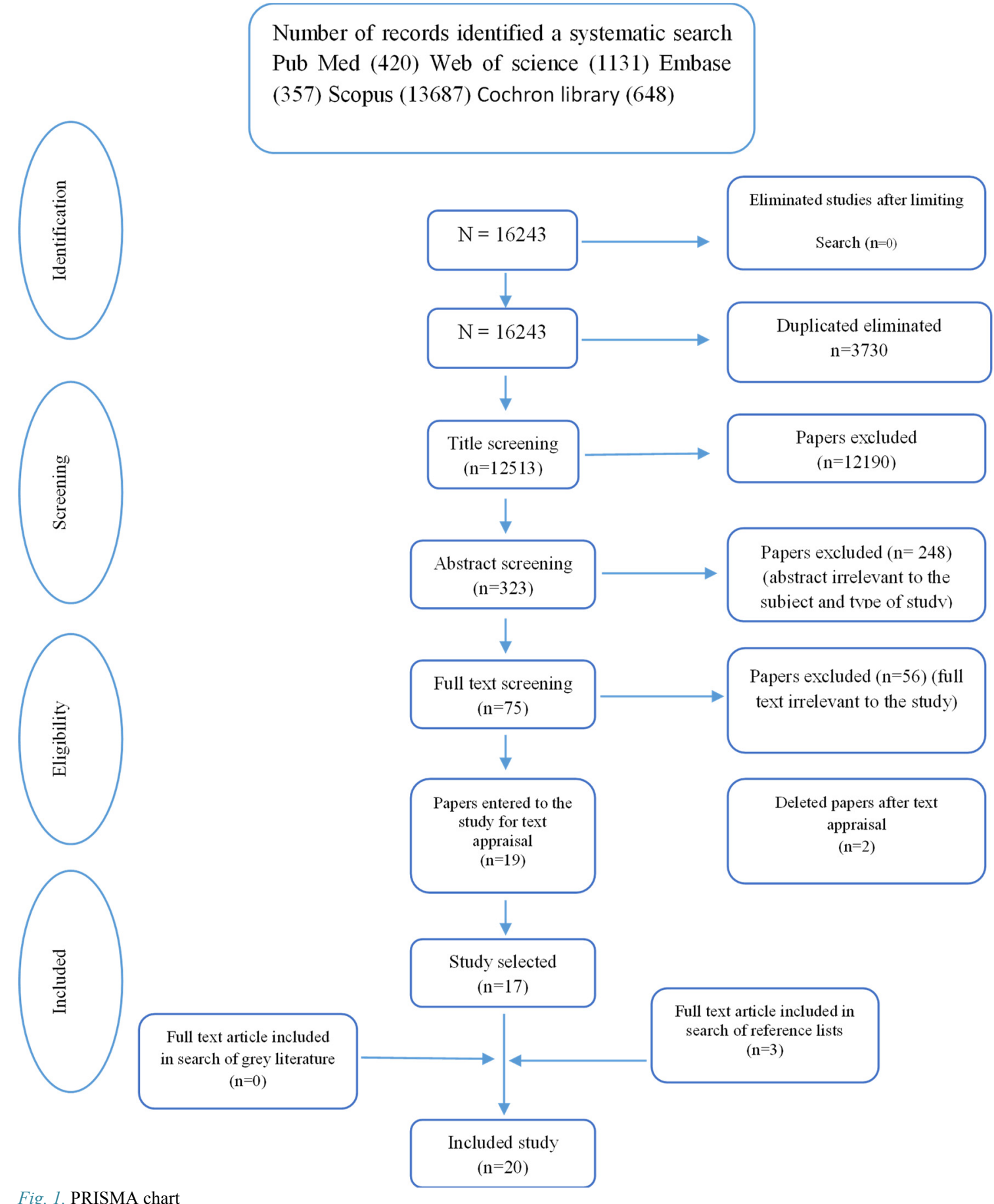

function), level and patient assessment (equivalent to stewardship function) were extracted (Table 1).

\section{Results}

20 models related to health service delivery for the elderly were extracted from the articles. These models are shown in Table 1.

1. Program of All-inclusive Care for the Elderly (PACE) $\mathrm{PACE}$ is a creative model for financing and long-term care

delivery in the United States. This model was launched in 1971 in San Francisco and was further developed until 1995. Its main purpose is the prevention of the use of unnecessary care in hospitals and nursing homes. This model provides services in clinics, daycare centers, hospitals, nursing homes, and patients' own homes. To enroll in this program, the applicant must be at least 55 years old, be approved by the state authorities as eligible, and live in the designated geographical area. 


\begin{tabular}{|c|c|c|c|c|c|c|c|c|c|c|}
\hline Model & Country/year & $\begin{array}{l}\text { Target } \\
\text { popula- } \\
\text { tion }\end{array}$ & Case manager & Team / members & $\begin{array}{l}\text { Entry (patient) } \\
\text { assessment }\end{array}$ & Services & Setting & Information system & Financing & Level \\
\hline PACE & USA/1971-1995 & +55 & Team & $\begin{array}{l}\text { interdisciplinary } \\
\text { team / primary care } \\
\text { physicians, nurse } \\
\text { practitioners, clinic } \\
\text { nurses, home health } \\
\text { nurses, social } \\
\text { workers, occupa- } \\
\text { tional and physical } \\
\text { therapists, dieti- } \\
\text { tians, health work- } \\
\text { ers, recreation ther- } \\
\text { apists, and trans- } \\
\text { portation workers }\end{array}$ & $\begin{array}{l}\text { Eligibility ac- } \\
\text { cording to } \\
\text { each state's } \\
\text { criteria such } \\
\text { as } \\
\text { live in the pro- } \\
\text { gram's defined } \\
\text { geographical }\end{array}$ & $\begin{array}{c}\text { Full spectrum of } \\
\text { medical, social, } \\
\text { and rehabilitative } \\
\text { services. }\end{array}$ & $\begin{array}{c}\text {-Hospital } \\
\text {-Home care } \\
\text {-Skilled Nursing facil- } \\
\text { ity care } \\
\text {-Hospice } \\
\text {-nursing home } \\
\text {-Community health } \\
\text { center }\end{array}$ & $\begin{array}{l}\text { Electronic record but } \\
\text { not system level clas- } \\
\text { sification system }\end{array}$ & $\begin{array}{l}\text { Medicare and Medicaid ( } \\
\text { Monthly capitation pay- } \\
\text { ments) }\end{array}$ & Local \\
\hline WPP & $\begin{array}{l}\text { USA/ } \\
2000\end{array}$ & +65 & Team & $\begin{array}{c}\text { Interdisciplinary } \\
\text { care management } \\
\text { team/ nurse, social } \\
\text { worker, and nurse } \\
\text { practitioner }\end{array}$ & - & $\begin{array}{l}\text { Like PACE ser- } \\
\text { vices }\end{array}$ & $\begin{array}{l}\text { Like PACE } \\
\text { Setting }\end{array}$ & - & Medicaid and Medicare & Local \\
\hline $\begin{array}{l}\text { High Inten- } \\
\text { sity care } \\
\text { manage- } \\
\text { ment model }\end{array}$ & USA/2002(publish) & +60 & $\begin{array}{l}\text { Yes but not } \\
\text { clear }\end{array}$ & $\begin{array}{l}\text { Periodic team } \\
\text { meetings }\end{array}$ & $\begin{array}{l}\text { Client assess- } \\
\text { ment and care } \\
\text { planning }\end{array}$ & $\begin{array}{c}\text { Acute and Long- } \\
\text { Term Care Ser- } \\
\text { vices }\end{array}$ & Home care & $\begin{array}{l}\text { Information system is } \\
\text { unclear }\end{array}$ & $\begin{array}{l}\text { Without waivers or fi- } \\
\text { nancing packages, and no } \\
\text { state or federal govern- } \\
\text { ment support. It relied on } \\
\text { agency and community } \\
\text { professional commitment }\end{array}$ & Local \\
\hline GRACE & USA/2002-2003 & +65 & - & $\begin{array}{l}\text { Interdisciplinary } \\
\text { team /geriatrician, } \\
\text { pharmacist, } \\
\text { physical therapist, } \\
\text { mental health so- } \\
\text { cial worker, and } \\
\text { community-based } \\
\text { services liaison }\end{array}$ & $\begin{array}{l}\text { Initial com- } \\
\text { prehensive } \\
\text { geriatric as- } \\
\text { sessment by } \\
\text { GRACE sup- } \\
\text { port team }\end{array}$ & $\begin{array}{c}\text {-Primary care } \\
\text {-specialty care } \\
\text {-Mental health } \\
\text {-Inpatient Geriatric } \\
\text { care services } \\
\text { - develop an indi- } \\
\text { vidualized } \\
\text { care plan }\end{array}$ & $\begin{array}{c}\text {-Hospital } \\
\text {-Home care } \\
\text { - Community-based } \\
\text { health centers }\end{array}$ & $\begin{array}{l}\text { An electronic medical } \\
\text { record and longitudi- } \\
\text { nal tracking system } \\
\text { (web-based care man- } \\
\text { agement tracking } \\
\text { tool) }\end{array}$ & $\begin{array}{l}\text {-grants from The Na- } \\
\text { tional Institute on Aging } \\
\text { and the Nina Mason Pul- } \\
\text { liam Charitable Trust, In- } \\
\text { dianapolis, Indiana. } \\
\text {-Approximately } 10 \% \text { of } \\
\text { the total cost of the inter- } \\
\text { vention was covered un- } \\
\text { der current Medicare re- } \\
\text { imbursement. }\end{array}$ & Local \\
\hline
\end{tabular}




\begin{tabular}{|c|c|c|c|c|c|c|c|c|c|c|}
\hline Model & Country/year & $\begin{array}{l}\text { Target } \\
\text { popula- } \\
\text { tion }\end{array}$ & Case manager & Team / members & $\begin{array}{c}\text { Entry (patient) } \\
\text { assessment }\end{array}$ & Services & Setting & Information system & Financing & Level \\
\hline $\begin{array}{r}\text { Medicaring } \\
\text { models }\end{array}$ & USA & +65 & - & $\begin{array}{l}\text { A multidiscipli- } \\
\text { nary team }\end{array}$ & $\begin{array}{l}\text { All individuals } \\
\text { over } 65 \text { who } \\
\text { are living with } \\
\text { disabilities } \\
\text { (more than } \\
\text { one ADL or } \\
\text { needing con- } \\
\text { stant supervi- } \\
\text { sion) and all } \\
\text { those over } 85 \\
\text { who opt for } \\
\text { coherent care } \\
\text { focused upon } \\
\text { their priorities }\end{array}$ & $\begin{array}{l}\text {-Long-term ser- } \\
\text { vices } \\
\text { - Supports ser- } \\
\text { vices such as } \\
\text { nutrition, hous- } \\
\text { ing, safety, } \\
\text { transportation, } \\
\text { personal care } \\
\text { - Medical and } \\
\text { nursing care } \\
\text { - Environmen- } \\
\text { tal services }\end{array}$ & $\begin{array}{c}\text {-Hospital } \\
\text {-Nursing home } \\
\text {-Assisted living fa- } \\
\text { cility } \\
\text {-(Focus on):home } \\
\text { care }\end{array}$ & $\begin{array}{l}\text { Locally managed } \\
\text { dashboards }\end{array}$ & $\begin{array}{l}\text { The financing model } \\
\text { owes much to the col- } \\
\text { laboration of } \\
\text { Dobson|Davanzo, } \\
\text { Inc., and to Jim Lee } \\
\text { of Altarum Institute. } \\
\text { This work was sup- } \\
\text { ported in part by a } \\
\text { grant from the Mil- } \\
\text { bank Fund for Reha- } \\
\text { bilitation. }\end{array}$ & Local \\
\hline $\begin{array}{r}\text { SAFECare } \\
\text { model }\end{array}$ & USA/2012-2013 & +65 & - & $\begin{array}{c}\text { Interprofessional } \\
\text { team /physician, } \\
\text { social worker, } \\
\text { pharmacist, pri- } \\
\text { mary RN, prac- } \\
\text { tice nurse, physi- } \\
\text { cal therapy and } \\
\text { occupational } \\
\text { therapy }\end{array}$ & SPICES tool & $\begin{array}{l}\text {-Post-acute care } \\
\text {-Palliative care } \\
\text { consultation } \\
\text {-PT and OT } \\
\text { consult }\end{array}$ & Hospital & $\begin{array}{l}\text { Electronic Health } \\
\text { Records }\end{array}$ & $\begin{array}{l}\text { Used existing ser- } \\
\text { vices }\end{array}$ & Local \\
\hline $\begin{array}{l}\text { The Illa- } \\
\text { warra Coor- } \\
\text { dinated Care } \\
\text { Trial }\end{array}$ & Australia/1997-2000 & +65 & $\begin{array}{l}\text { General prac- } \\
\text { titioner }\end{array}$ & - & $\begin{array}{l}\text { A formal as- } \\
\text { sessment of all } \\
\text { participants }\end{array}$ & $\begin{array}{c}\text {-Public services } \\
\text {-Private ser- } \\
\text { vices } \\
\text { - Medical (GP } \\
\text { and Specialist) } \\
\text {-Health and so- } \\
\text { cial services } \\
\text {-Pharmaceutical } \\
\text { and hospital } \\
\text { services. }\end{array}$ & $\begin{array}{c}\text { - Hospital } \\
\text {-Home care } \\
\text { - Residential cen- } \\
\text { ters }\end{array}$ & $\begin{array}{l}\text { The development of } \\
\text { an independent linked } \\
\text { information system }\end{array}$ & $\begin{array}{l}\text { Create a pool of funds } \\
\text { from four main } \\
\text { sources: the Health } \\
\text { Insurance Commis- } \\
\text { sion (HIC) ; the Illa- } \\
\text { warra Area Health } \\
\text { Service (IAHS); the } \\
\text { Home and Commu- } \\
\text { nity Care (HACC) } \\
\text { program; and the De- } \\
\text { partment of Veterans' } \\
\text { Affairs (DVA). }\end{array}$ & Local \\
\hline
\end{tabular}


Health service delivery models for elderly people

\begin{tabular}{|c|c|c|c|c|c|c|c|c|c|c|}
\hline Model & Country/year & $\begin{array}{c}\text { Target } \\
\text { population }\end{array}$ & Case manager & Team / members & $\begin{array}{c}\text { Entry (patient) } \\
\text { assessment }\end{array}$ & Services & Setting & $\begin{array}{c}\text { Information } \\
\text { system }\end{array}$ & Financing & Level \\
\hline $\begin{array}{l}\text { The British } \\
\text { Columbia } \\
\text { Continuing } \\
\text { Care system }\end{array}$ & Canada/ & +65 & $\begin{array}{l}\text { Yes but not } \\
\text { clear }\end{array}$ & $\begin{array}{l}\text { Quick Response } \\
\text { Teams }\end{array}$ & $\begin{array}{c}\text { Standardized, } \\
\text { system level } \\
\text { (5-level) as- } \\
\text { sessment and } \\
\text { care authoriza- } \\
\text { tion. }\end{array}$ & $\begin{array}{c}\text { A wide range of health } \\
\text { and social services such } \\
\text { as home care nursing, } \\
\text { community rehabilita- } \\
\text { tion, home support ser- } \\
\text { vices, adult day care } \\
\text { services and group } \\
\text { homes, mental health, } \\
\text { housing, continuing } \\
\text { Care, programs (respite } \\
\text { care) } \\
\text { Adult Day Care pro- } \\
\text { grams, Meal Programs }\end{array}$ & $\begin{array}{c}\text {-Hospital } \\
\text {-Home Care } \\
\text {-Nursing Home } \\
\text { - Respite care } \\
\text { - Long term care fa- } \\
\text { cilities } \\
\text {-Residential care } \\
\text { - Adult Day Care } \\
\text { center }\end{array}$ & $\begin{array}{l}\text { An integrated } \\
\text { information } \\
\text { system }\end{array}$ & $\begin{array}{l}\text { A single funding enve- } \\
\text { lope }\end{array}$ & national \\
\hline SIPA & $\begin{array}{l}\text { Canada } \\
/ 1995\end{array}$ & - & $\begin{array}{l}\text { Nurses or so- } \\
\text { cial workers }\end{array}$ & $\begin{array}{c}\text { Interdisciplinary } \\
\text { team / Case man- } \\
\text { agers, Commu- } \\
\text { nity nurses, So- } \\
\text { cial workers, Oc- } \\
\text { cupational thera- } \\
\text { pists, Physiother- } \\
\text { apists, Home- } \\
\text { makers, Staff } \\
\text { family physi- } \\
\text { cians, Consultant } \\
\text { pharmacists, } \\
\text { Community or- } \\
\text { ganizers }\end{array}$ & $\begin{array}{c}\text { Functional au- } \\
\text { tonomy Meas- } \\
\text { urement Sys- } \\
\text { tem (SMAF) } \\
\text { scale }\end{array}$ & $\begin{array}{l}\text { Primary and secondary } \\
\text { medical and social ser- } \\
\text { vices, prevention, reha- } \\
\text { bilitation, medication, } \\
\text { technical aids and long- } \\
\text { term care }\end{array}$ & $\begin{array}{c}\text {-Nursing homes } \\
\text {-Home care } \\
\text {-Day centers } \\
\text { - Hospitals } \\
\text { - Long-term care in- } \\
\text { stitutions }\end{array}$ & $\begin{array}{c}\text { Electronic In- } \\
\text { formation Sys- } \\
\text { tem }\end{array}$ & $\begin{array}{l}\text { Capitation payment } \\
\text { (not implemented in } \\
\text { the demonstration pro- } \\
\text { ject) }\end{array}$ & Local \\
\hline PRISMA & Canada/ 1997-2003 & +65 & $\begin{array}{c}\text { Yes } \\
\text { but not clear }\end{array}$ & $\begin{array}{l}\text { multidisciplinary } \\
\text { team }\end{array}$ & SMAF scale & $\begin{array}{c}\text {-Long term care } \\
\text {-hospital and rehabilita- } \\
\text { tion } \\
\text {-Nursing care } \\
\text {-Social services } \\
\text {-The individualized ser- } \\
\text { vice plan }\end{array}$ & $\begin{array}{c}\text {-Home care } \\
\text {-Hospital } \\
\text {-Long term care } \\
\text { Institutions } \\
\text {-Voluntary Agen- } \\
\text { cies (Meals-on- } \\
\text { Wheels) } \\
\text {-Day Center } \\
\text {-Nursing home } \\
\text {-... }\end{array}$ & $\begin{array}{l}\text { A computerized } \\
\text { clinical chart } \\
\text { for communi- } \\
\text { cating between } \\
\text { institutions for } \\
\text { client monitor- } \\
\text { ing purposes. }\end{array}$ & $\begin{array}{l}\text { The Canadian Health } \\
\text { Services Research } \\
\text { Foundation and } \\
\text { budget negotiations } \\
\text { between partner or- } \\
\text { ganizations }\end{array}$ & Local \\
\hline
\end{tabular}

6 http://mjiri.iums.ac.ir

Med J Islam Repub Iran. 2021 (10 Feb); 35:21. 


\begin{tabular}{|c|c|c|c|c|c|c|c|c|c|c|}
\hline Model & Country/year & $\begin{array}{c}\text { Target } \\
\text { population }\end{array}$ & Case manager & Team / members & $\begin{array}{c}\text { Entry (patient) } \\
\text { assessment }\end{array}$ & Services & Setting & Information system & Financing & Level \\
\hline COPA & France $/ 2006$ & +65 & $\begin{array}{l}\text { Gerontology } \\
\text { nurses }\end{array}$ & $\begin{array}{c}\text { multidisciplinary } \\
\text { primary care } \\
\text { team (Include: } \\
\text { case manager, } \\
\text { PCP, psycholo- } \\
\text { gists and Other } \\
\text { professionals }\end{array}$ & $\begin{array}{l}\text { Inter RAI } \\
\text { MDS-HC }\end{array}$ & $\begin{array}{l}\text { - Primary medi- } \\
\text { cal care } \\
\text {-Specialized } \\
\text { care } \\
\text {-Long term care } \\
\text {-Social services }\end{array}$ & $\begin{array}{c}\text { - Home care } \\
\text {-Hospital } \\
\text {-Nursing home } \\
\text {-.... }\end{array}$ & $\begin{array}{l}\text { A shared clinical infor- } \\
\text { mation system }\end{array}$ & $\begin{array}{l}\text { The regional funding } \\
\text { agency }\end{array}$ & local \\
\hline $\begin{array}{l}\text { Hong Kong } \\
\text { Model }\end{array}$ & Hong Kong / 2000 & +60 & $\begin{array}{l}\text { A social } \\
\text { worker plus a } \\
\text { registered } \\
\text { nurse }\end{array}$ & - & $\begin{array}{l}\text { Hong Kong } \\
\text { version of the } \\
\text { Minimum } \\
\text { Data Set- } \\
\text { Home Care } \\
\text { (MDS-HC) }\end{array}$ & $\begin{array}{c}\text {-Home visits } \\
\text { and telephone } \\
\text { consultations } \\
\text {-Health educa- } \\
\text { tional programs } \\
\text { - Integrated } \\
\text { health and social } \\
\text { services } \\
\text {-Day rehabilita- } \\
\text { tive program } \\
\text {-Community } \\
\text { support services }\end{array}$ & $\begin{array}{c}\text {-Home care } \\
\text { - Respite hospital } \\
\text { Care } \\
\text { - Day care center } \\
\text { - Day hospital } \\
\text {-Clinic }\end{array}$ & $\begin{array}{l}\text { Use of an electronic } \\
\text { information system }\end{array}$ & $\begin{array}{l}\text { Used available ser- } \\
\text { vices and research } \\
\text { funds }\end{array}$ & Local \\
\hline Rovereto & $\begin{array}{l}\text { Italy/ } \\
1995\end{array}$ & +65 & $\begin{array}{l}\text { Two Case } \\
\text { managers. For } \\
\text { home: general } \\
\text { practitioner/ } \\
\text { for hospital: } \\
\text { Community } \\
\text { geriatric } \\
\text { evaluation unit }\end{array}$ & $\begin{array}{l}\text { Multidisciplinary } \\
\text { team }\end{array}$ & $\begin{array}{l}\text { Standardized } \\
\text { assessment and } \\
\text { care planning }\end{array}$ & $\begin{array}{l}\text { Integrated social } \\
\text { and medical care }\end{array}$ & $\begin{array}{c}\text {-Home care } \\
\text {-Day hospital } \\
\text {-Hospital in home } \\
\text {-Nursing home }\end{array}$ & $\begin{array}{l}\text { Electronic information } \\
\text { system }\end{array}$ & $\begin{array}{l}\text { Used available ser- } \\
\text { vices and research } \\
\text { funds }\end{array}$ & Local \\
\hline $\begin{array}{l}\text { The Silver } \\
\text { Network } \\
\text { project }\end{array}$ & $\begin{array}{c}\text { Italy/ } \\
1997-1998\end{array}$ & - & $\begin{array}{l}\text { Geriatric } \\
\text { nurses }\end{array}$ & $\begin{array}{l}\text { Interdisciplinary } \\
\text { teams }\end{array}$ & $\begin{array}{l}\text { The Minimum } \\
\text { Data Set for } \\
\text { Home Care } \\
\text { (MDS-HC) }\end{array}$ & $\begin{array}{l}\text { All services are } \\
\text { provided in an } \\
\text { integrated fash- } \\
\text { ion (health and } \\
\text { social services) }\end{array}$ & $\begin{array}{l}\text {-Home care } \\
\text {-Nursing home } \\
\text {-Day hospital }\end{array}$ & $\begin{array}{l}\text { A database including } \\
\text { the Minimum Data Set } \\
\text { (MDS) and infor- } \\
\text { mation on all drugs } \\
\text { used by each patient. }\end{array}$ & $\begin{array}{l}\text { Pfizer Italy Silver Net- } \\
\text { work Project }\end{array}$ & Local \\
\hline DGIP & Netherlands & +70 & - & $\begin{array}{c}\text { Multidisciplinary } \\
\text { Team/ geriatric } \\
\text { specialist nurse, } \\
\text { GP and a geriatri- } \\
\text { cian }\end{array}$ & $\begin{array}{l}\text { EASYcare in- } \\
\text { strument }\end{array}$ & $\begin{array}{l}\text { Individualized, } \\
\text { community- } \\
\text { based } \\
\text { integrated treat- } \\
\text { ment plan } \\
\end{array}$ & $\begin{array}{l}\text {-Home care } \\
\text {-Nursing home }\end{array}$ & $\begin{array}{l}\text { General Practice's In- } \\
\text { formation System } \\
\text { (Huisartsen Informatie } \\
\text { System) }\end{array}$ & $\begin{array}{l}\text { Radboud University } \\
\text { Nijmegen Medical } \\
\text { Center }\end{array}$ & Local \\
\hline
\end{tabular}




\begin{tabular}{|c|c|c|c|c|c|c|c|c|c|c|}
\hline Model & Country/year & $\begin{array}{c}\text { Target } \\
\text { population }\end{array}$ & Case manager & Team / members & $\begin{array}{l}\text { Entry (patient) } \\
\text { assessment }\end{array}$ & Services & Setting & Information system & Financing & Level \\
\hline WICM & Netherland & +75 & $\begin{array}{l}\text { Two case man- } \\
\text { ager. } \\
\text { The geriatric } \\
\text { nurse practi- } \\
\text { tioner for el- } \\
\text { derly with sin- } \\
\text { gle needs / A } \\
\text { secondary line } \\
\text { geriatric nurs- } \\
\text { ing specialist } \\
\text { for multiple or } \\
\text { complex needs }\end{array}$ & $\begin{array}{l}\text { Multidisciplinary } \\
\text { Team/general } \\
\text { practitioner and } \\
\text { case manager and } \\
\text { other profession- } \\
\text { als }\end{array}$ & $\begin{array}{l}\text { the Groningen } \\
\text { Frailty Indica- } \\
\text { tor (GFI) and } \\
\text { EASY care tool }\end{array}$ & $\begin{array}{c}\text { - Health and so- } \\
\text { cial services } \\
\text {-Community ser- } \\
\text { vices } \\
\text {-Health profes- } \\
\text { sional } \\
\text {-Other (Care, } \\
\text { Cure, Mental } \\
\text { health, paramedi- } \\
\text { cal, } \\
\text { Welfare, hous- } \\
\text { ing) } \\
\text {-Individualized } \\
\text { service plan }\end{array}$ & $\begin{array}{c}\text {-Hospital } \\
\text {-Home care } \\
\text {-Nursing home } \\
\text { - Respite care }\end{array}$ & $\begin{array}{l}\text { - A chain computeriza- } \\
\text { tion system }\end{array}$ & $\begin{array}{c}\text { The Netherlands Or- } \\
\text { ganization for Health } \\
\text { Research and Develop- } \\
\text { ment and health insurer } \\
\text { CZ }\end{array}$ & Local \\
\hline $\begin{array}{l}\text { The Care- } \\
\text { Well-primary } \\
\text { care program }\end{array}$ & $\begin{array}{l}\text { Netherlands/ } \\
2008\end{array}$ & +70 & $\begin{array}{l}\text { Community } \\
\text { nurse or the } \\
\text { gerontological } \\
\text { social }\end{array}$ & $\begin{array}{l}\text { Multidisciplinary } \\
\text { teams / GP, the } \\
\text { community nurse, } \\
\text { an elderly care } \\
\text { physician and a } \\
\text { gerontological so- } \\
\text { cial worker }\end{array}$ & $\begin{array}{c}\text { The Easy Care- } \\
\text { TOS }\end{array}$ & Primary care & $\begin{array}{l}\text {-Residential home } \\
\text { - Nursing home } \\
\text {-Hospital } \\
\text {-Home visit }\end{array}$ & $\begin{array}{l}\text { web-based health and } \\
\text { information welfare } \\
\text { portal (ZWIP) }\end{array}$ & $\begin{array}{l}\text { The Dutch Organiza- } \\
\text { tion for Health Re- } \\
\text { search and Develop- } \\
\text { ment }\end{array}$ & Local \\
\hline PoC & $\begin{array}{l}\text { Netherlands/ } \\
2008-2010\end{array}$ & +70 & Practice nurse & $\begin{array}{c}\text { Interdisciplinary } \\
\text { team (including a } \\
\text { general practi- } \\
\text { tioner, practice } \\
\text { nurse, } \\
\text { occupational and } \\
\text { physical thera- } \\
\text { pists, a pharmacist } \\
\text { or a geriatrician, } \\
\text { nurse specialist, } \\
\text { expert in technol- } \\
\text { ogy and re- } \\
\text { searcher }\end{array}$ & GFI & $\begin{array}{l}\text {-Primary care } \\
\text {-Disability pre- } \\
\text { vention program } \\
\text {-Social support }\end{array}$ & -Home visit & $\begin{array}{l}\text { The Centre for Data } \\
\text { and Information Man- } \\
\text { agement (MEMIC) }\end{array}$ & $\begin{array}{l}\text { The Dutch National } \\
\text { Care for the Elderly } \\
\text { Program by the Nether- } \\
\text { lands Organization for } \\
\text { Health Research and } \\
\text { Development }\end{array}$ & National \\
\hline $\begin{array}{r}\text { Embrace } \\
\text { model }\end{array}$ & $\begin{array}{l}\text { Netherlands/ } \\
2012-2013\end{array}$ & +75 & $\begin{array}{l}\text { Two case man- } \\
\text { agers (district } \\
\text { nurse and so- } \\
\text { cial worker) }\end{array}$ & $\begin{array}{c}\text { A multidiscipli- } \\
\text { nary } \\
\text { Elderly Care } \\
\text { Team / general } \\
\text { practitioner, an el- } \\
\text { derly care physi- } \\
\text { cian, and two case } \\
\text { managers (district } \\
\text { nurce and conial }\end{array}$ & $\begin{array}{c}\text {-Annual screen- } \\
\text { ing with self-re- } \\
\text { port question- } \\
\text { naire. } \\
\text {-The Groningen } \\
\text { Frailty Indica- } \\
\text { tor } \\
\text { (GFI) }\end{array}$ & $\begin{array}{l}\text {-Comprehensive, } \\
\text { Patient centered, } \\
\text { proactive, pre- } \\
\text { ventive care } \\
\text { - Support ser- } \\
\text { vices }\end{array}$ & $\begin{array}{c}\text {-Hospital } \\
\text {-Nursing home } \\
\text {-Home health care }\end{array}$ & $\begin{array}{l}\text { An Electronic Elderly } \\
\text { Record System(Clinical } \\
\text { information systems) }\end{array}$ & $\begin{array}{l}\text { Netherlands Organiza- } \\
\text { tion for Health Re- } \\
\text { search and Develop- } \\
\text { ment. }\end{array}$ & Local \\
\hline
\end{tabular}




\begin{tabular}{|c|c|c|c|c|c|c|c|c|c|c|}
\hline Model & Country/year & $\begin{array}{c}\text { Target } \\
\text { population }\end{array}$ & Case manager & Team / members & $\begin{array}{l}\text { Entry (patient) } \\
\text { assessment }\end{array}$ & Services & Setting & Information system & Financing & Level \\
\hline $\begin{array}{l}\text { IHMS } \\
\end{array}$ & Spain- Austria/ 2018 & - & - & $\begin{array}{l}\text {-Primary health } \\
\text { team } \\
\text {-Interagency } \\
\text { community team } \\
\text {-Chronic disease } \\
\text { specialist team }\end{array}$ & New ICT tool & $\begin{array}{l}\text {-Health and so- } \\
\text { cial services } \\
\text {-Palliative care } \\
\text {-Personalize } \\
\text { health diagno- } \\
\text { sis, treatment, } \\
\text { prevention, and } \\
\text { health promo- } \\
\text { tion }\end{array}$ & $\begin{array}{l}\text {-Person centered } \\
\text { health care } \\
\text {-Home based care }\end{array}$ & $\begin{array}{c}\text { - local IHMS Data } \\
\text { Banks } \\
\text {-IHMS National and } \\
\text { international IHMS } \\
\text { Data Bank }\end{array}$ & $\begin{array}{l}\text { Shared capital re- } \\
\text { sources }\end{array}$ & National \\
\hline
\end{tabular}


This program receives monthly per capita payments from Medicare and Medicaid insurances and covers a wider range of benefits than Medicare and Medicaid do. PACE is a comprehensive care model and delivers a full range of medical, social, and rehabilitation services. This model uses an interdisciplinary team to manage treatment and integration of primary and specialized medical care (14).

\section{The Wisconsin Partnership Program (WPP)}

The WPP was designed in the United States in 2000 and is a variation of the PACE model. The WPP is designed to provide more flexibility for dual-eligible older people, enabling them to use primary care physicians instead of physicians employed at PACE. Its purpose is to merge the resources of these programs in order to improve quality services and avoid duplications. In this model, a team of nurses, nurse practitioners, and social workers provides case management (15).

\section{High-Intensity Care Management model}

This model was designed in the United States, and its target group was 60-year-old and older patients. In this model, very intense care management was used to improve the integration of acute care and long-term care. In this regard, the model attempted to use a case manager supervised by a geriatrician to supplement the management of the existing care at home. The frail elderly (the intervention group) received enhanced clinical service management in addition to traditional care management. This model attempted to create cooperation-based integration without financial or regulatory incentives, so it was not able to bring about adequate change in the care system or better outcomes for the elderly (16).

\section{Geriatric Resources for Assessment and Care of El- ders (GRACE)}

GRACE is a primary care model for poor seniors and their PCPs that was implemented in 2002-2003 in Indiana, USA. The goals of this model were to improve the quality of care for the elderly in order to optimize their health and functional status, reducing excessive use of health care, and preventing long-term care at nursing homes. The GRACE catalyst is a support team. After registration, this team conducts a full assessment of the elderly. This team then meets with the interdisciplinary team to make an individualized care program. The support team then meets with PCPs to discuss and revise the developed program, thus designing the best treatment plan for the elderly (17).

\section{Medicaring Model}

This is a comprehensive model that was designed in the United States to provide higher-quality services to the frail elderly under Medicare at a lower cost. The Elderly are 65 or older and are either dependent on others in two or more activities, are almost permanently dependent on others due to cognitive disorders, or are people 85 years old and older who are aware of their physiological limitations. The emphasis in this model is on the delivery of cost-effective services at home. After identifying the disabled elderly and the identification of the priorities of each elderly person by a multidisciplinary team, an individualized long-term care program and support services (such as nutrition, transportation, safety, and housing) is designed for each frail elderly (18).

\section{Systems Addressing Frail Elder Care (SAFE care) model}

This program was designed and implemented from 2012 to 2013 in a hospital in the USA. The aim of this model was to identify risk among patients 65 years old and older and to use an interdisciplinary approach to treat them. After the patients were screened by a geriatric nurse and the high-risk patients were identified, each team member made an accurate assessment of the high-risk patients according to their specialization. Team members then had a meeting moderated by a physician in order to reach an agreement. After identifying the risks, recommendations were made for each patient to deal with the risks and improve the condition of each elderly person (19).

\section{The Illawarra Coordinated Care Trial}

This model was one of the nine trials conducted between 1997 and 2000 in Australia. This trial was conducted to coordinate the care of people 65 and older with the risk of falls or multifaceted medical or social difficulties that require several services from more than one provider. Care was coordinated by 16 care coordinators in collaboration with the GP (case manager). He/she controlled different medical aspects of the paitents' care, while the care coordinator arranged availability to other services. After a systematic evaluation was made by the coordinator to identify the care programs the patient needed, the tailored patient service package was finally designed. These services were mainly provided by community care service providers who had access to different services (20).

\section{The British Columbia Continuing Care system}

This model is an integrated delivery system created in British Columbia, Canada. The system has an entry point and includes all the main components of long-term care and home care services for the frail elderly. In this model, a wider range of different services such as home care, rehabilitation, etc., is provided. For most senior citizens, the services are provided in one of two ways. The first way involves the implementation of home or community-based services through an external services provider. The second way is to provide home care by nurses and therapists through the Community Home Care Nursing and Rehabilitation Program. Either Assessors or the Case Manager Places both home and community-based patients in one of five distinct levels of care, and each elderly person receives services according to their needs (21).

\section{SIPA: a System of Integrated Care for the Frail Elderly}

SIPA is a system based primary care designed in 1995 in Canada. The purpose of designing this model is to resolve the problems of the frail elderly, provide comprehensive care, and ensure its continuity. SIPA uses an integrated case 
management model to organize and provide the most community-based services. All eligible seniors are registered in SIPA after evaluation. Treatment is provided by an interdisciplinary team including health and social care professionals including a family physician. The team is responsible for assessing patient needs, planning, and providing services. The interdisciplinary team tries to improve patient performance and minimize the inappropriate acute and long-term use of institutions. This model delivers a full range of health (all levels) and social services (22).

10. The Program of Research to Integrate the Services for the Maintenance of Autonomy (PRISMA) Model

PRISMA is a Canadian creative co-ordination-type Integrated Service Delivery System. The purpose of this model is to improve the continuity and increase the efficiency and effectiveness of services, especially for the elderly and disabled people. Cooperation among institutions forms the core of PRISMA. In this model, a single entry point is used, and the elderly are evaluated using the available tools. The case manager is responsible for conducting a thorough assessment of patient needs and development to provide services to them. The case manager is working with a multidisciplinary team to personalize the treatment plan for each senior. In addition to hospitals and rehabilitation services, long-term service centers, volunteer organizations, home care, and nursing homes are among the centers providing services to the elderly in this model. In addition to hospitals and rehabilitation services, services are also provided to the elderly by long-term service centers, volunteer organizations, home care, and nursing homes (23).

\section{The COPA model}

COPA is a model based on scientific evidence implemented in 2006 in Paris, France. The purpose of this model is to balance the available services and the elderly needs in order to reduce the excessive use of health services. COPA focuses on a specific high-risk group (very weak elderly people living in a particular service area) and coordinates treatment at two levels, within primary care and between primary care and specialized care. In this model, the role of primary care physicians is strengthened and includes patient recruitment and developing treatment plans. COPA also uses a multidisciplinary primary care team, including different professionals, especially case managers. This team works with primary care physicians to assess the elderly and provide care for them. COPA organizes integrated primary care and specialized care through geriatricians for visiting the elderly in their homes and direct hospitalization by primary care physicians (24).

\section{Hong Kong model}

This program was launched in 2000 for the cost-benefit analysis of a case management program. The seniors were classified into two groups: the control and the intervention group. In the control group, the elders received standard services. In the intervention group, the elders were first visited by a case manager at their home and were counseled over the phone. After a comprehensive evaluation, they were divided into four groups of severe impairment, moderate impairment, mild impairment, and without impairment based on their treatment status and received care plans based on their condition. Health education and support programs were also provided for the elderly in the intervention group, alongside treatment programs (25).

\section{Roverto model}

In the early $1990 \mathrm{~s}$, the health agency of Rovereto, Italy, created a wide range of health services for older people. They included a hospital geriatric evaluation unit, a skilled nursing facility, and a home health agency; nevertheless, these components did not have any cooperation with each other. In 1995, an integrated treatment model composed of a case manager, a multidisciplinary team, a community geriatric evaluation unit, and a general practitioner was created. All persons aged 65 and above were identified. After the initial assessment, the case manager reported the condition of each elderly person to the geriatric evaluation unit, and together they determined the services the senior was eligible for. Finally, with the agreement of the client's general practitioner, suitable medical and social services for each elderly person were provided (26).

\section{The Silver Network project}

This program was designed and implemented in Italy in 1997 and 1998. This model integrates all services of health agencies or municipalities. It has a community geriatric evaluation unit (CGEU), which plays a key role in the process of determining the qualification of the elderly to use long-term care. Health and social service delivery to the frail elderly is coordinated by a case manager who uses, MDS-HC Assessment Tool. Based on the data derived from this evaluation tool for each patient, researchers find out which of the elderly is eligible for home care (27).

\section{The Dutch Geriatric Intervention Program (DGIP)}

DGIP is a multidisciplinary community intervention model designed in the Netherlands, which includes nurse home visits for elderly patients. This model uses a multidisciplinary team, including a geriatrics nurse, a GP, and a geriatrician. The Easycare assessment tool is used to assess the condition of patients. The GP refers to elders with cognitive, mood, behavior, mobility, and nutrition impairments. A geriatric specialist nurse implements a guidelinebased intervention. During the intervention, the nurse regularly consults the referring treatment physician and a geriatrician. A wide multidimensional assessment is performed for each patient, and an integrated and individualized treatment plan is developed based on each patient's evaluation results (28).

\section{The Walcheren Integrated Care Model (WICM)}

This model is part of the National Elderly Care Program of the Netherlands. WICM is a comprehensive, integrated model for the identification and assessment of needs and the assignment and evaluation of care for disabled elders who live independently. The patients aged 75 and above are identified by a GP using the GFI tool. Elderly people with 
GFI $\geq 4$ are recognized as disabled and transfer to a case manager. The case manager then organizes meetings with the elderly to assess their needs using the Easycare instrument. The goals are organized in consultation with the patients and their providers. The case manager then describes the results of the assessments in the form of a proposed individualized care plan. Then the proposal is discussed in a meeting with the presence of various experts and led by a GP. In this meeting, the multidisciplinary care program is approved, care actions and paths are discussed, and agreements are made on the dispatch and tasks of all concerned parties. Finally, an individualized care plan is design for each elderly patient (29).

\section{The CareWell-primary care program}

This model was designed and implemented in 2008 in the eastern region of the Netherlands. Its purpose is to avoid functional weakness, improve quality of life, and decrease or delay admissions in hospitals and nursing homes. After the necessary assessments, the frail elderly enter the model cycle. General practitioners work with one or two multidisciplinary teams to ensure the integration of treatment, care, and welfare of the elderly. Based on the assessment of the condition of the elderly and their personal needs, a care plan is designed for them. This model uses supportive elements such as consultation with geriatric experts to facilitate and provide care plans (30).

\section{Prevention of Care (POC)}

POC is an interdisciplinary model that was designed in the years 2008-2010 in the Netherlands for primary care for the frail elderly. The core aim of this model is to prevent the disability of the elderly. The frail elderly receive their personalized care program from the multidisciplinary care team after the initial assessment. A GP and a practice nurse (case manager) comprise the core team, which can be expanded by other professionals such as an occupational and physical therapist. After screening, seniors aged 70 and above (with GFI score $\geq 5$ ) are assessed. After the initial assessment, an initial action plan is designed based on the patient's evaluation results, history, risk factors, and individual needs. After finalization, the action plan, an individualized care plan will be implemented for each elderly person. Finally, the care plans are evaluated and followed up (31).

\section{Embrace model}

This model was designed and implemented in the Netherlands in 2012 and 2013. Its goal is to provide comprehensive, patient-centered, and preventive services to support all older people $(+75)$. This model is based on the chronic care model and the population health management model. Frail elderly are enrolled in the program by GPs. After necessary assessment, the elderly are divided into three groups of robust, frail, and complex care needs. The robust elderly are asked to follow an independent supportive self-management program focusing on health maintenance for as long as possible. Frail seniors and those with multifaceted care needs receive individual support services (appropriate to their condition) from a case manager. They are advised to adhere to the self-management and prevention program (32).

\section{The Integrated Health care Management System} (IHMS)

This model was designed by researchers from Austria and Spain in collaboration with a team of experts from different disciplines. This is a united home- and communitybased health care management system that uses the latest technologies to personalize services. In this model, different teams, such as a primary health team, interagency community team, and a chronic disease specialist team, are used to provide services to the elderly suffering from chronic diseases. After assessing the condition of the elderly and with the support of information systems and the use of new tools, health services appropriate to the patient's needs are provided. This model delivers a wide range of diagnostic, treatment, palliative, social, and health promotion services, and its focus is mainly on home care (33).

\section{Discussion}

Regarding the increasing population of the world's elderly and the need to pay special attention to the medical needs of this group, countries with high elderly populations are expected to use appropriate models to provide services to the elderly, but the models that were found are only limited to the USA ( 6 models), the Netherlands (5 models), Canada (3 models), Italy ( 2 models), France (1 model), Australia (1 model), Hong Kong (1 model) and another model (which was Designed by researchers from Austria and Spain). Most of these models have been designed in the past 30 years and mostly at the level of a city or a province, and have differences and similarities that are debatable.

Most models use an evaluation system or evaluation tool to identify frail elderly and enter them into the model process. Due to the complexity of the health problems of the elderly, having multiple chronic diseases, etc. organic structures are used in most models. Therefore, most of them use a case manager who is in cooperation with a multi-disciplinary team in their structure. The case manager is usually a doctor, a nurse, and in some cases, a social worker who is responsible for monitoring and following up treatment of the elderly. Most models have a case manager but Embrace and Roverto uses two case managers. Some models, such as IHMS, Medicare, Grace, SAFE Care, and DGIP, do not mention a case manager.

Most models use an interdisciplinary or multidisciplinary team that depending on the characteristics of each model, the purpose, and services that it provides can include different disciplines such as medicine, nursing, physiotherapy, social work, etc. this team is responsible for planning, treatment, and tending to issues of the elderly. Using data from the Assessment of the elderly, as well as information systems support, team members try to work together in the best possible way and monitor the improvement of the elderly.

These models usually provide integrated care for the elderly, although depending on their purpose and focus, the range of services they provide is different in different cases . For example, GRACE, POCE, and Carewell primary care 
focus more on primary care. Models such as SIPA, PACE, and British Colombia provide more comprehensive services such as treatment, rehabilitation, and social services. Also, due to the insufficient potential of hospitals, most models have used alternative methods such as home care to provide services to the elderly.

The found models were using a variety of financing methods. In most models, financing was mainly done by the executive university or a research organization, and they provided services to the elderly using existing resources (manpower and equipment). But the SIPA, PACE, WPP, IHMS, COPA, PRISMA, British Colombia, Illawarra models mainly use stronger and more sustainable financing.

In general, models can be classified into two levels: small models (local level) and large models (national level). Most of the models are small models, but the IHMS, POC, and British Colombia are considered to be large. Small models can be implemented at the national or provincial / state level without any major change in policy or law and generally do not require additional financial resources. As models get larger, they may need to make more changes to community health laws and policies. In larger models, there is a need for more cooperation and coordination between the available elements, which makes them more difficult to implement and monitor.

\section{Conclusion}

Due to the complex needs of the elderly and the high cost of their care and treatment, it is necessary to use more community-based models and flexible structure based on case management. Although in local models, the source of funding is mainly the source of research or other available resources and services, these models need sustainable financial resources such as health and social insurance to continue. It seems that most models are not dominant and are not used at the national level. In order for smaller models to become standard models at the national level, they need to be reviewed and evaluated by large-scale policymakers and managers and then be implemented in other areas after the necessary reforms.

\section{Acknowledgment}

The researcher would like to thank Kerman University of Medical Sciences, for their financing.

\section{Conflict of Interests}

The authors declare that they have no competing interests.

\section{References}

1. WHO. 10 facts on ageing and the life course. World Health Organization; 2012.

2. United Nations. World Population Ageing 2017: Highlights. New York: Department of Economic and Social Affairs, United Nations. 2017.

3. Gobbens RJ, Luijkx KG, Wijnen-Sponselee MT, Schols JM. Toward a conceptual definition of frail community dwelling older people. Nurs Outlook. 2010;58(2):76-86.

4. Van Kan GA, Sinclair A, Andrieu S, Rikkert MO, Gambassi G, Vellas $\mathrm{B}$, et al. The geriatric minimum data set for clinical trials (GMDS). J
Nutr Health Aging. 2008;12(3):197-200.

5. O'Connor C. Visioning the future: health care for the elderly. Arizona State University; 2006.

6. Hafez G, Bagchi K, Mahaini R. Caring for the elderly: a report on the status of care for the elderly in the Eastern Mediterranean Region. East Mediterr Health J. 2000;6(4):636-42.

7. WHO. World report on ageing and health. World Health Organization; 2015.

8. Rechel B, Doyle Y, Grundy E, McKee M, World Health Organization. How can health systems respond to population ageing. Copenhagen: WHO Regional Office for Europe; 2009.

9. Zon MW. The Netherlands Organisation for Health Research and Development: National programme of care for the elderly. The Hague:Ministry of Health, Welfare and Sports, the Netherlands; 2008:54.

10. Boult C, Wieland GD. Comprehensive primary care for older patients with multiple chronic conditions: "Nobody rushes you through". JAMA. 2010;304(17):1936-1943.

11. Reuben DB. Meeting the needs of disabled older persons: can the fragments be pieced together? J Gerontol A Biol Sci Med Sci. 2006;61(4):365-366.

12. Clarfield AM, Bergman H, Kane R. Fragmentation of care for frail older people-an international problem: Experience from three countries: israel, canada, and the united states. J Am Geriatr Soc. 2001;49(12):1714-1721.

13. CASP. Critical Appraisal Skills Programme (CASP). http://www. casp- uk. net/ casp- tools- checklists (accessed Oct 2017).

14. Eng C, Pedulla J, Eleazer GP, McCann R, Fox N. Program of Allinclusive Care for the Elderly (PACE): an innovative model of integrated geriatric care and financing. $\mathrm{J}$ Am Geriatr Soc. 1997;45(2):223-32.

15. Kane RL, Homyak P, Bershadsky B, Lum YS. Consumer responses to the Wisconsin Partnership Program for Elderly Persons: a variation on the PACE Model. J Gerontol A Biol Sci Med Sci. 2002;57(4):2508 .

16. Applebaum R, Straker J, Mehdizadeh S, Warshaw G, Gothelf E. Using high-intensity care management to integrate acute and long-term care services: substitute for large scale system reform? Care Manag J. 2002;3(3):113-9.

17. Counsell SR, Callahan CM, Buttar AB, Clark DO, Frank KI. Geriatric Resources for Assessment and Care of Elders (GRACE): A new model of primary care for low-income seniors. J Am Geriatr Soc. 2006;54(7):1136-41.

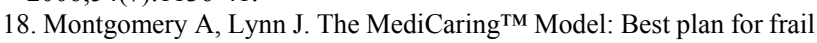
elders in the longevity era. Public Policy Aging Rep. 2014;24(3):1127.

19. Ansryan LZ, Aronow HU, Borenstein JE, Mena V, Haus F, Palmer K, et al. Systems addressing frail elder care: Description of a successful model. J Nurs Adm. 2018;48(1):11-7.

20. Perkins D, Owen A, Cromwell D, Adamson L, Eagar K, Quinset K, et al. The Illawarra Coordinated Care Trial: better outcomes with existing resources? Aust Health Rev. 2001;24(2):172-8.

21. Hollander MJ, Pallan P. The British Columbia continuing care system: service delivery and resource planning. Aging Clin Exp Res. 1995;7(2):94-109.

22. Béland F, Bergman H, Lebel P, Clarfield AM, Tousignant P, Contandriopoulos AP, et al. A system of integrated care for older persons with disabilities in Canada: results from a randomized controlled trial. J Gerontol A Biol Sci Med Sci. 2006;61(4):367-73.

23. Hébert R, Durand PJ, Dubuc N, Tourigny A, Group P. PRISMA: a new model of integrated service delivery for the frail older people in Canada. Int J Integr Care. 2003;3.

24. Vedel I, De Stampa M, Bergman H, Ankri J, Cassou B, Mauriat C, et al. A novel model of integrated care for the elderly: COPA, Coordination of Professional Care for the Elderly. Aging Clin Exp Res. 2009;21(6):414-23.

25. Leung AC, Liu CP, Chow NW, Chi I. Cost-benefit analysis of a case management project for the community-dwelling frail elderly in Hong Kong. J Appl Gerontol. 2004;23(1):70-85.

26. Bernabei R, Landi F, Gambassi G, Sgadari A, Zuccala G, Mor V, et al. Randomised trial of impact of model of integrated care and case management for older people living in the community. BMJ. 1998;316(7141): 1348.

27. Landi F, Gambassi G, Pola R, Tabaccanti S, Cavinato T, Carbonin P, et al. Impact of integrated home care services on hospital use. J Am 
Geriatr Soc. 1999;47(12):1430-4

28. Melis RJ, van Eijken MI, Borm GF, Wensing M, Adang E, van de Lisdonk EH, et al. The design of the Dutch EASYcare study: a randomised controlled trial on the effectiveness of a problem-based community intervention model for frail elderly people. BMC Health Serv Res. 2005;5(1):65.

29. Fabbricotti IN, Janse B, Looman WM, De Kuijper R, Van Wijngaarden JDH, Reiffers A. Integrated care for frail elderly compared to usual care: a study protocol of a quasi-experiment on the effects on the frail elderly, their caregivers, health professionals and health care costs. BMC Geriatr. 2013;13(1):31.

30. Ruikes FG, Meys AR, van de Wetering G, Akkermans RP, van Gaal BG, Zuidema SU, et al. The CareWell-primary care program: design of a cluster controlled trial and process evaluation of a complex intervention targeting community-dwelling frail elderly. BMC Fam Pract. 2012;13(1):115.

31. Daniels R, van Rossum E, Metzelthin S, Sipers W, Habets H, Hobma $\mathrm{S}$, et al. A disability prevention programme for community-dwelling frail older persons. Clin Rehabil. 2011;25(11):963-74.

32. Spoorenberg SL, Uittenbroek RJ, Middel B, Kremer BP, Reijneveld SA, Wynia K. Embrace, a model for integrated elderly care: study protocol of a randomized controlled trial on the effectiveness regarding patient outcomes, service use, costs, and quality of care. BMC Geriatr. 2013;13(1):62.

33. Gandarillas MÁ, Goswami N. Merging current health care trends: innovative perspective in aging care. Clin Interv Aging. 2018;13:2083. 